\title{
钯催化氧杂降冰片烯与三氟硼酸钾盐的不对称开环反应
}

\author{
陶萍芳 $\dagger$ 黄 俊 ${ }^{\dagger}, a, b$ 刘玉钊 ${ }^{a}$ 韦光明 $a$ 王艺菲 ${ }^{a}$ \\ 韦贤生 ${ }^{a}$ 黄国保*, 李秀英*,a \\ ( ${ }^{a}$ 玉林师范学院化学与食品科学学院 广西农产资源化学与生物技术重点实验室 广西玉林 537000) \\ ( ${ }^{b}$ 南宁师范大学化学与材料学院 南宁 530001)
}

\author{
摘要 开发了一种新型高效的钯催化的三氟硼酸钾盐与氧杂降冰片烯的不对称开环反应的方法. 在温和的条件下, 可 \\ 得到相应的顺-2-芳基-1,2-二氢荥-1-醇产物, 其产率良好, 目标产物对映体选择性较高. \\ 关键词 氧杂降冰片烯; 三氟钾硼酸盐; 不对称开环反应
}

\section{Palladium-Catalyzed Asymmetric Ring-Opening Reactions of Oxabenzonorbornadienes with Potassium Trifluoroborate Salts}

\author{
Tao, Pingfang ${ }^{\dagger, a} \quad$ Huang, Jun ${ }^{\dagger, a, b}$ \\ Liu, Yuzhao ${ }^{a}$ \\ Wei, Guangming ${ }^{a}$ \\ Wang, Yifei ${ }^{a}$ \\ Wei, Xiansheng ${ }^{a}$ \\ Huang, Guobao*,a \\ Li, Xiuying ${ }^{*, a}$ \\ ( ${ }^{a}$ Guangxi Key Laboratory of Agricultural Resources Chemistry and Biotechnology, College of Chemistry and Food Science, \\ Yulin Normal University, Yulin, Guangxi 537000) \\ ( ${ }^{b}$ College of Chemistry and Materials, Nanning Normal University, Nanning 530001)
}

\begin{abstract}
A new, versatile and highly efficient palladium-catalyzed asymmetric ring-opening reaction of oxabenzonorbornadienes with a wide range of potassium trifluoroborate salts was developed. The corresponding cis-2-aryl-1,2dihydronaphthalen-1-ol products were obtained in good yields with moderate to good enantioselectivities under mild conditions.

Keywords oxabenzonorbornadiene; trifluoroborate salt; asymmetric ring-opening reaction
\end{abstract}

\section{Introduction}

The synthesis of substituted 1,2-dihydronaphthalenes is a theme of ongoing interest because these compounds possess a wide range of biological activities, ${ }^{[1]}$ and they are also important intermediates in organic synthesis. ${ }^{[2]}$ Among the available methods for hydronaphthalenes synthesis, the transition metal-catalyzed asymmetric ringopening (ARO) reactions of hetero-bicyclic alkenes with various carbanion and heteroatom nucleophiles are valuable processes, which have been intensively investigated. Many metal catalysts such as $\mathrm{Fe},{ }^{[3]} \mathrm{Ni},{ }^{[4]} \mathrm{Cu},{ }^{[5]} \mathrm{Ru}^{[6]} \mathrm{Rh},{ }^{[7]}$ $\mathrm{Pd}{ }^{[8]} \mathrm{Pt}^{[9]}$ and $\operatorname{Ir}^{[10]}$ were explored for the ring-opening of oxa- and aza-bicyclic alkenes.
How to find a group of novel nucleophiles as the aryl source to react with oxabicyclic alkenes is a long-term challenge to the chemists. Various aryl donators such as aryl halides, ${ }^{[1]]}$ organometallic reagents, ${ }^{[12]}$ arylboronic acids $^{[13]}$ and arylsulfinate salts ${ }^{[14]}$ had been used in the ARO reactions. To the best of our knowledge, the study referring to the reaction of aryl halides with oxabenzonorbornadiene is relatively rare because of the low activity of these carbanion nucleophiles. On the contrary, high active organometallic reagents may incur some side effects to give the ring-opening product in low yields. ${ }^{[12 a]}$ In recent years, arylsulfinate salts due to the stability and low toxicity, have been employed as effective carbanion nucleophiles in the ring-opening reactions of oxabicyclic alkenes

\footnotetext{
* Corresponding authors. E-mail: 1zjx0915@163.com; 1xya8401@163.com

Received December 22, 2019; revised March 11, 2020; published online March 31, 2020.

Project supported by the National Natural Science Foundation of China (No. 21961042), the Natural Science Foundation of Guangxi Province (No. 2018GXNSFAA294064) and the Open Fund of Guangxi Key Laboratory of Agricultural Resources Chemistry and Biotechnology Research Grant (No. KF04). 国家自然科学基金(No.21961042)、广西自然科学基金(No. 2018GXNSFAA294064)及广西农产资源化学与生物技术重点实验室开放基金(No. KF04)资 助项目.

共同第一作者(These authors contributed equally to this work).
} 
to afford the corresponding products, but with low enantioselectivities. Arylboronic acids are appropriate reagents in this type of ARO reactions due to their low cost, remarkable stability and ease of handling. However, arylboronic acids still have some drawbacks, such as having high nucleophile equivalence and low reactivity in the air atmosphere. Nevertheless, these results gave us good references.

Potassium trifluoroborate salts are a kind of ideal reagents in organic chemistry, for example, the construction of $\mathrm{C}-\mathrm{C}$ bonds. ${ }^{[15]}$ Compared with boronic acids, potassium trifluoroborate salts not only own similar chemical properties, but also are more convenient and stable. ${ }^{[16]}$ To our surprise, so far there is no report on the ring opening reaction of oxybenzodiene with potassium trifluoborate as the substrate. Herein a novel ARO addition of potassium trifluoroborate salts to oxabicyclic alkenes in the presence of palladium complex to afford the corresponding products of cis-2-aryl-1,2-dihydronapthalene-1-ols in high yields (up to $98 \%$ ) with moderate to good enantioselectivities (up to $98 \% e e$ ) is reported. cis-Configuration, not transconfiguration, of the product was obtained through X-ray diffraction analysis.

\section{Results and discussion}

To explore the ring opening reaction, preliminary optimization of the reaction conditions was carried out using oxabenzonorbornadiene (1a) and potassium phenyltrifluoroborate (2a) as model substrates in toluene/water $(V: V=5: 1)$ at room temperature. When 1 a reacted with 2a in the presence of $5 \mathrm{~mol} \% \mathrm{Pd}(\mathrm{OAc})_{2}$ and $10 \mathrm{~mol} \%$ DPPP under nitrogen atmosphere, desired ring-opened product 3a was obtained in 25\% isolated yield after $24 \mathrm{~h}$ (Table 1, Entry 1). Encouraged by this result, several chiral ligands were screened for the ring-opening reaction of $\mathbf{1 a}$ under similar conditions, including $(S, S)$-Me-DuPhos, $(S)$-DM-SegPhos, $(R)-(S)$-PPF-PtBu $2,(R)$-BINAP and $(S)$-PipPhos. The results indicated that $(R)$-BINAP was the most effective ligand among the ligands screened for this reaction compared with the others (Table 1, Entries $2 \sim 6$ ), and $3 \mathbf{a}$ was afforded in $90 \%$ yield with $55 \%$ ee (Table 1 , Entry 6). Meanwhile, the catalyst loading also had a significant impact. The results indicated that $5 \mathrm{~mol} \%$ $\mathrm{Pd}(\mathrm{OAc})_{2}$ with $10 \mathrm{~mol} \%(R)$-BINAP would be a more suitable catalyst loading in terms of the yield and enantioselectivity, whereas increasing or decreasing the amount of catalyst loading improved neither the yield nor ee value of 3a (Table 1, Entries 7 9). Similar yield and enantioselectivity were obtained whether in a nitrogen or air atmosphere (Table 1, Entry 10). In addition, the effect of solvents was investigated. A mixture of water and $\mathrm{CH}_{3} \mathrm{CN}$ was totally ineffective in this reaction (Table 1, Entry 11). When the reaction was carried out in a mixed solvent of water with tetrahydrofuran (THF), expected product 3a was afforded in only $20 \%$ yield with $30 \%$ ee (Table 1 , Entry 12). It was observed that the reaction in a mixed solvent of water with protic solvents $\mathrm{MeOH}$ gave satisfa-
Table 1 Optimization of reaction conditions for the ARO reaction $^{a}$

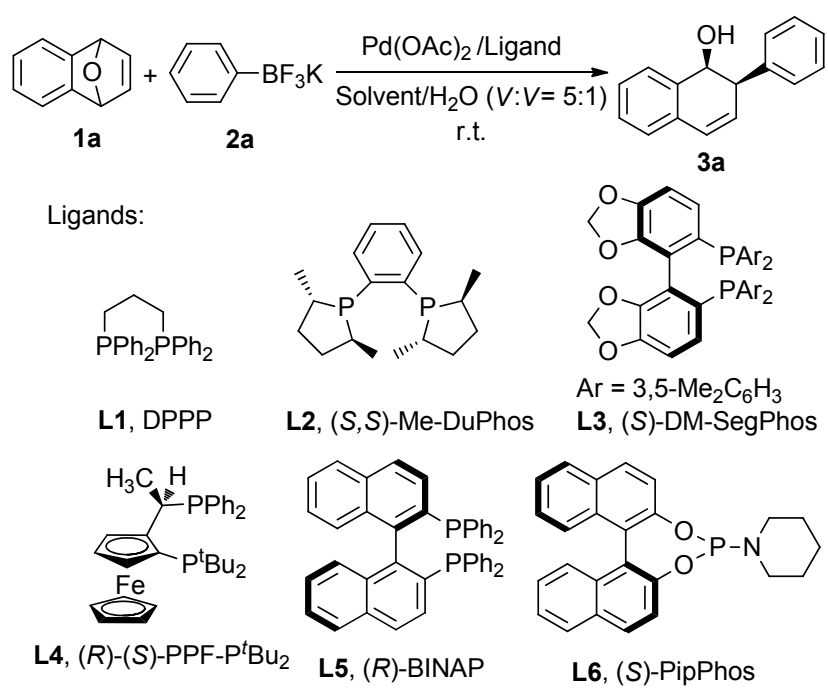

\begin{tabular}{|c|c|c|c|c|c|c|}
\hline Entry & Solvent & $\begin{array}{c}\mathrm{Pd}(\mathrm{OAc})_{2} / \\
\mathrm{mol} \%\end{array}$ & Ligand & Time/h & Yield/\% & $e e^{b} / \%$ \\
\hline 1 & Toluene & 5.0 & L1 & 24 & 25 & - \\
\hline 2 & Toluene & 5.0 & $\mathbf{L 2}$ & 7 & 49 & 35 \\
\hline 3 & Toluene & 5.0 & L3 & 7 & 60 & 21 \\
\hline 4 & Toluene & 5.0 & L4 & 24 & $\mathrm{NR}^{c}$ & - \\
\hline 5 & Toluene & 5.0 & L5 & 0.5 & 90 & 55 \\
\hline 6 & Toluene & 5.0 & L6 & 24 & $\mathrm{NR}^{c}$ & - \\
\hline 7 & Toluene & 2.5 & L5 & 1 & 80 & 55 \\
\hline 8 & Toluene & 1.0 & L5 & 2 & 71 & 55 \\
\hline 9 & Toluene & 10.0 & L5 & 0.5 & 92 & 54 \\
\hline $10^{d}$ & Toluene & 5.0 & L5 & 0.5 & 91 & 55 \\
\hline $11^{d}$ & $\mathrm{MeCN}$ & 5.0 & L5 & 24 & $\mathrm{NR}^{c}$ & - \\
\hline $12^{d}$ & THF & 5.0 & L5 & 24 & 20 & 30 \\
\hline $13^{d}$ & $\mathrm{MeOH}$ & 5.0 & L5 & 22 & 30 & 76 \\
\hline $14^{d}$ & $\mathrm{CH}_{2} \mathrm{Cl}_{2}$ & 5.0 & L5 & 3 & 85 & 45 \\
\hline $15^{d}$ & $\mathrm{CHCl}_{3}$ & 5.0 & L5 & 3 & 80 & 50 \\
\hline $16^{d}$ & DCE & 5.0 & L5 & 1 & 81 & 52 \\
\hline $17^{d, e}$ & Toluene & 5.0 & L5 & 5 & 72 & 58 \\
\hline $18^{d, f}$ & Toluene & 5.0 & L5 & 5 & 85 & 42 \\
\hline
\end{tabular}

${ }^{a}$ Reaction conditions: 1a $(0.2 \mathrm{mmol}), \mathbf{2 a}\left(1.2\right.$ equiv.), $\mathrm{Pd}(\mathrm{OAc})_{2}$ and ligand (10 mol\%) in solvent and $\mathrm{H}_{2} \mathrm{O}(V: V=5: 1,3 \mathrm{~mL}$ in total) under nitrogen at room temperature. ${ }^{b}$ Determined by HPLC with a chiralcel OD-H column. ${ }^{c}$ NR: no reaction. ${ }^{d}$ Carried out in air. ${ }^{e}$ The reaction at $0{ }^{\circ} \mathrm{C} .{ }^{f}$ The reaction at $50{ }^{\circ} \mathrm{C}$.

ctory enantioselectivity (up to $76 \%$ ee) but poor conversion (Table 1, Entry 13). Moderate to good yields and enantioselectivities were obtained when a mixed solvent of water with $\mathrm{CH}_{2} \mathrm{Cl}_{2}, \mathrm{CHCl}_{3}$ and dichloroethane (DCE) were respectively employed as the solvents (Table 1, Entries $14 \sim 16$ ). Importantly, the study on solvent effect indicated that the reaction worked very well in a mixed solvent of water with the aromatic solvent of toluene showed the best combination of reactivity and enantioselectivity among all solvents tested ( $91 \%$ yield, $55 \%$ ee). However, satisfactory results had not been achieved when the impact of reaction temperature was investigated. The reaction run at $0{ }^{\circ} \mathrm{C}$ gave a diminished yield and slightly improved ee (Table 1, 
Entry 17). Raising the temperature to $50{ }^{\circ} \mathrm{C}$ somewhat decreased the yield and ee even after a prolonged reaction time (Table 1, Entry 18).

Next, with the optimized reaction conditions identified, the scope of potassium trifluoroborate salts was explored for this reaction to evaluate their effect on the reactivity and enantioselectivity (Table 2). From Table 2, it can be seen that the structures of potassium trifluoroborate salts have significant impact on the reactivity and enantioselectivities. In general, potassium trifluoroborate salts with electron-donating substituents on the phenyl rings gave higher yields but lower enantioselectivities than those with electron-withdrawing groups (Table 2, Entries $2 \sim 9$ ). When it came to the position property of monosubstituted on the phenyl rings of potassium trifluoroborate salts, it had little effect on the reactivity, and the ring-opening of 1a with parasubstituted potassium trifluoroborate salts offerd better enantioselectivities than those with meta- and ortho-substituted potassium trifluoroborate salts (Table 2, Entries 2, 3, 5 7). It is noteworthy that the addition of alkyl-potassium trifluoroborate salt to $\mathbf{1 a}$ was found to give the corresponding product $\mathbf{3 j}$ successfully just prolonged the reaction time to $3 \mathrm{~h}$ (Table 2, Entry 10). Potassium trifluoroborate salts containing heterocycle were employed in the ring-opening of 1a, the results was also satisfactory (Table 2, Entry 11). Thiophene-2-potassium trifluoroborate salt reacted with 1a and 3k was obtained in high yield (95\% yield) with modest enantioselectivity $(71 \% e e)$.

Table 2 Ring-opening of oxabenzonorbornadiene 1a with various potassium trifluoroborate salts ${ }^{a}$

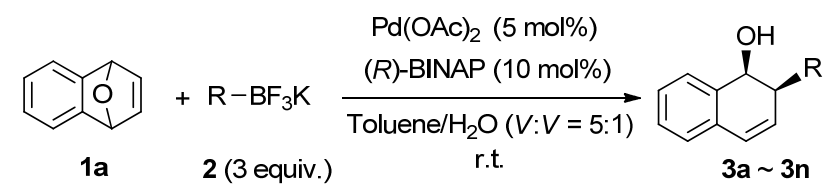

\begin{tabular}{clcccc}
\hline Entry & \multicolumn{1}{c}{$\mathrm{R}$} & Product & Time/h & Yield $/ \%$ & $e e^{b} / \%$ \\
\hline 1 & $\mathrm{C}_{6} \mathrm{H}_{5}$ & $\mathbf{3 a}$ & 0.5 & 91 & 55 \\
2 & $4-\mathrm{CH}_{3} \mathrm{C}_{6} \mathrm{H}_{4}$ & $\mathbf{3 b}$ & 0.5 & 94 & 62 \\
3 & $2-\mathrm{CH}_{3} \mathrm{C}_{6} \mathrm{H}_{4}$ & $\mathbf{3 c}$ & 0.5 & 92 & 20 \\
4 & $4-\mathrm{BuC}_{6} \mathrm{H}_{4}$ & $\mathbf{3 d}$ & 0.5 & 95 & 36 \\
5 & $2-\mathrm{CH}_{3} \mathrm{OC}_{6} \mathrm{H}_{4}$ & $\mathbf{3 e}$ & 0.5 & 90 & 2 \\
6 & $3-\mathrm{CH}_{3} \mathrm{OC}_{6} \mathrm{H}_{4}$ & $\mathbf{3 f}$ & 0.5 & 91 & 56 \\
7 & $4-\mathrm{CH}_{3} \mathrm{OC}_{6} \mathrm{H}_{4}$ & $\mathbf{3 g}$ & 0.5 & 98 & 51 \\
8 & $4-\mathrm{BrC}_{6} \mathrm{H}_{4}$ & $\mathbf{3 h}$ & 0.5 & 84 & 64 \\
9 & $4-\mathrm{FC}_{6} \mathrm{H}_{4}$ & $\mathbf{3 i}$ & 0.5 & 84 & 60 \\
10 & $\mathrm{CH}_{3}$ & $\mathbf{3 j}$ & 3 & 81 & 56 \\
11 & $2-\mathrm{Thienyl}_{3}$ & $\mathbf{3 k}$ & 0.5 & 95 & 71 \\
\hline
\end{tabular}

${ }^{a}$ Reaction conditions: 1a $(0.2 \mathrm{mmol}), 2$ (1.2 equiv.), $\mathrm{Pd}(\mathrm{OAc})_{2}(5 \mathrm{~mol} \%)$, and (R)-BINAP (10 mol\%) in toluene and $\mathrm{H}_{2} \mathrm{O}(V: V=5: 1,3 \mathrm{~mL}$ in total) in air at room temperature. ${ }^{b}$ Determined by HPLC with a chiralcel OD-H column.

The molecular configuration of $\mathbf{3 d}$ was unambiguously confirmed by X-ray diffraction analysis (Figure 1). The single crystal was obtained by solvent evaporation from a mixture of $\mathrm{CHCl}_{3}$ and hexane. The configuration of $\mathbf{3 d}$ was assigned as $(1 S, 2 R)$ and confirmed as the 1,2-cis- configu- ration. $^{[13 \mathrm{e}]}$
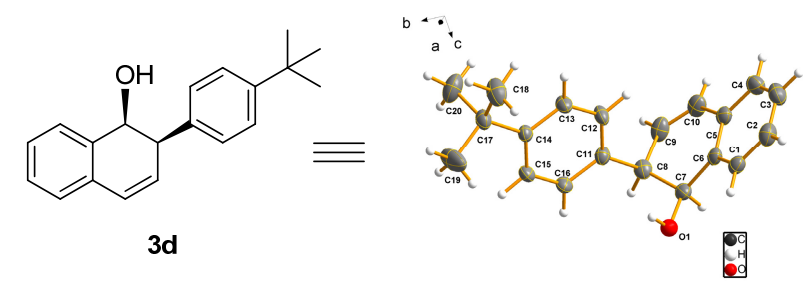

Figure 1 X-ray structure of the product 3d

The substrate scope of oxabenzonorbornadiene derivatives was further evaluated, and the results were compiled in Table 3. From Table 3, besides 1a, it can be seen that the reaction of various oxabenzonorbornadienes with potassium trifluoroborate salts proceeded smoothly to generate the anticipated ring-opening products in high yields (up to $93 \%$ ) with good to excellent enantioselectivity (up to 97\% ee). Furthermore, the influence of different potassium trifluoroborate salts on the reaction of each substrate is similar to that of substrate 1a, potassium trifluoroborate salts with electron-donating substituents on the phenyl rings gave higher yields but lower enantioselectivities than those with electron-withdrawing groups. The excellent performance of this catalyst system is highlighted by the reaction of 1b with potassium cyclopropyl trifluoroboranuide, which furnished product $\mathbf{4 a}$ in excellent yield $(90 \%)$ with remarkable enantioselectivity (up to $97 \%$ ee) (Table 3, Entry 2).

Table 3 Ring-opening of oxabenzonorbornadiene $\mathbf{1 b}$ and $\mathbf{1 c}$ with various potassium trifluoroborate salts ${ }^{a}$

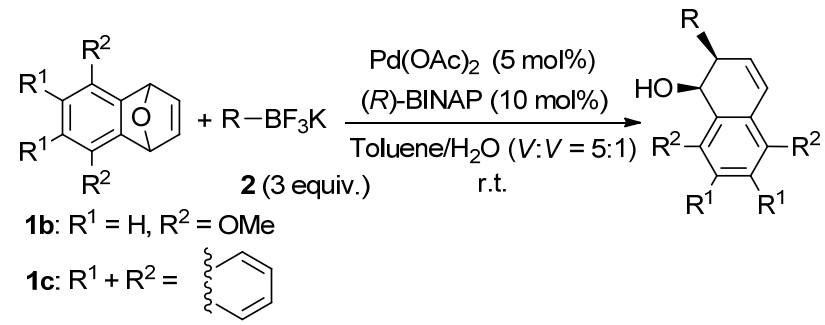

\begin{tabular}{cclccc}
\hline Entry & Substrate & \multicolumn{1}{c}{$\mathrm{R}$} & Product & Yield $/ \%$ & $e e^{b} / \%$ \\
\hline 1 & 1b & Cyclopropyl & $\mathbf{4 a}$ & 90 & 97 \\
2 & $\mathbf{1 b}$ & 4- $-\mathrm{FC}_{6} \mathrm{H}_{4}$ & $\mathbf{4 b}$ & 84 & 52 \\
3 & $\mathbf{1 b}$ & $4-\mathrm{CH}_{3} \mathrm{C}_{6} \mathrm{H}_{4}$ & $\mathbf{4 c}$ & 91 & 51 \\
4 & $\mathbf{1 c}$ & $\mathrm{C}_{6} \mathrm{H}_{5}$ & $\mathbf{5 a}$ & 85 & 55 \\
5 & $\mathbf{1 c}$ & Cyclopropyl & $\mathbf{5 b}$ & 90 & 97 \\
6 & $\mathbf{1 c}$ & 4- $\mathrm{FC}_{6} \mathrm{H}_{4}$ & $\mathbf{5 c}$ & 84 & 68 \\
7 & $\mathbf{1 c}$ & 4- $\mathrm{CH}_{3} \mathrm{C}_{6} \mathrm{H}_{4}$ & $\mathbf{5 d}$ & 91 & 65 \\
8 & $\mathbf{1 c}$ & 2-Thienyl & $\mathbf{5 e}$ & 93 & 87 \\
\hline
\end{tabular}

${ }^{a}$ Reaction conditions: 1b, 1c $(0.2 \mathrm{mmol}), 2$ (1.2 equiv.), $\mathrm{Pd}(\mathrm{OAc})_{2}(5 \mathrm{~mol} \%)$, and (R)-BINAP $(10 \mathrm{~mol} \%)$ in toluene and $\mathrm{H}_{2} \mathrm{O}(V: V=5: 1,3 \mathrm{~mL}$ in total $)$ in air at room temperature. ${ }^{b}$ Determined by HPLC with a chiralcel OD-H column.

Based upon the experimental observations and literatures, ${ }^{[17]}$ a proposed mechanism for the palladium(II)catalyzed asymmetric ring-opening of potassium phenyltrifluoroborate to oxaenzonornadiene $\mathbf{3 a}$ is summarized in Scheme 1 . The catalytic cycle would be initiated by the 
coordination of $\mathrm{Pd}(\mathrm{OAc})_{2}$ and $(R)$-BINAP to form the active chiral palladium complex $\mathbf{A}$, which leads to intermediate $\mathbf{B}$ after addition of potassium phenyltrifluoroborate (2a) ${ }^{[18]} \mathbf{B}$ is followed by insertion of azabenzonorbornadiene (1a) into the carbon-palladium bond from the exo side and produce intermediate C. $\beta$-Elimination of oxygen results in opening the furyl ring to give the ring-opened intermediate D which follows by the hydrolysis to liberate ring-opened product 3a ${ }^{[19]}$ Meanwhile, the palladium species $\mathbf{A}$ is regenerated to promote the next catalytic cycle.

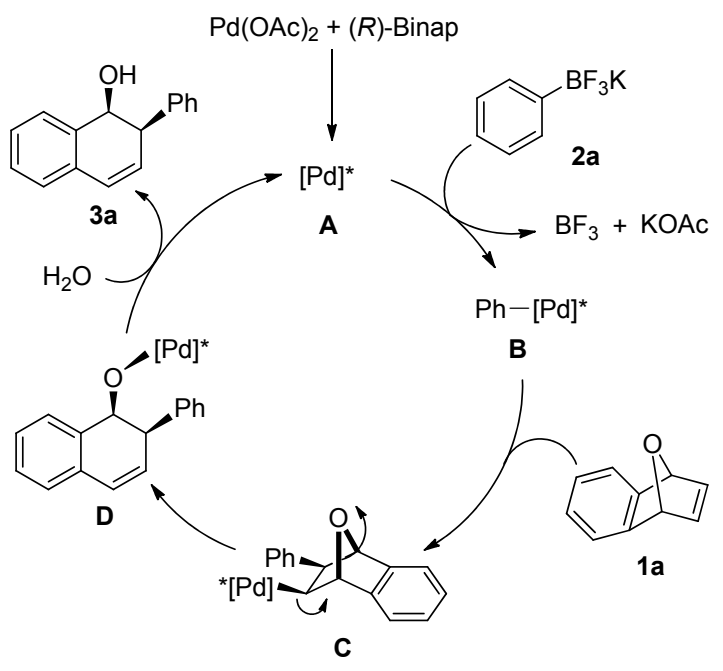

Scheme 1 Proposed mechanism

\section{Conclusions}

In summary, a new palladium(II)-catalyzed asymmetric ring-opening reaction of oxabenzonorbornadienes with potassium trifluoroborate salts was successfully developed. $\mathrm{Pd}(\mathrm{OAc})_{2}$ and $(R)$-BINAP were used as the catalyst system in this reaction. A practical and efficient approach was provided to synthesize the optically active cis-2-aryl-1,2dihydronaphthalen-1-ol derivatives in high yields with moderate to good enantioselectivities under mild conditions. Further investigations on the applications of palladium catalysts in the asymmetric ring-opening are in progress in our laboratory and will be reported.

\section{Experimental section}

\subsection{General information}

${ }^{1} \mathrm{H}$ NMR, ${ }^{13} \mathrm{C}$ NMR and ${ }^{19} \mathrm{~F}$ NMR spectra were recorded on a Bruker Avance nuclear magnetic resonance spectrometer at $400 / 500 \mathrm{MHz}$ and $100 / 125 \mathrm{MHz}$ at room temperature in $\mathrm{CDCl}_{3}$, respectively. HRMS (ion trap) were recorded by Q EXACTIVE mass spectrometry using APCI or ESI. The HPLC analysis was performed on a Waters Alliance e2695 high performance liquid chromatography with a Chiralcel OD-H column or Chiralpak AD-H column. Unless otherwise indicated, all reagents were purchased from commercial suppliers and used without further purification. Toluene was used without any pretreatment.
All flasks were flame-dried under a stream of nitrogen and cooled to room temperature before use. Oxabenzonorbornadienes $1 \mathbf{a} \sim \mathbf{1 c}$ were prepared according to the literature procedures. ${ }^{[20]}$

\subsection{General procedures for the products}

All experiments were carried out under an air atmosphere. Palladium diacetate (2.2 $\mathrm{mg}, 5 \mathrm{~mol} \%)$ and $(R)$-BINAP (12.4 mg, $10 \mathrm{~mol} \%$ ) were added to a $10 \mathrm{~mL}$ round-bottomed flask, followed by the addition of toluene/ $\mathrm{H}_{2} \mathrm{O}(V: V=5: 1,3 \mathrm{~mL}$ in total $)$. After the mixture was stirred for about $5 \mathrm{~min}$, oxabenzonorbornadienes 1a 1c $(0.2 \mathrm{mmol})$ and potassium trifluoroborate (1.2 equiv., $0.24 \mathrm{mmol}$ ) were put into the reaction system. The resulting mixture was stirred at room temperature until completion monitored by thin-layer chromatography (TLC). The solvent was removed in vacuo, and the crude mixture was then purified by column chromatography on silica gel (silica gel, $200 \sim 300 \mathrm{mesh}$ ) to afford the target product.

$\left(1 S^{*}, 2 R^{*}\right)$-2-Phenyl-1,2-dihydronaphthalen-1-ol (3a): Colorless oil ( $40.0 \mathrm{mg}, 90 \%$ yield). $R_{\mathrm{f}}=0.25$ on silica gel (ethyl acetate/petroleum ether, $V: V=1: 20$ ). The ee was determined to be $55 \%$ using HPLC analysis on a Chiralcel OD-H column (hexane/2-propanol, $V: V=90: 10,1.0$ $\mathrm{mL} / \mathrm{min}, \lambda=254 \mathrm{~nm}$ ). Retention times were 7.474 (major) and 11.708 (minor) min. ${ }^{1} \mathrm{H}$ NMR $\left(500 \mathrm{MHz}, \mathrm{CDCl}_{3}\right) \delta$ : $7.41 \sim 7.28(\mathrm{~m}, 8 \mathrm{H}), 7.22(\mathrm{~d}, J=7.2 \mathrm{~Hz}, 1 \mathrm{H}), 6.76(\mathrm{dd}, J=$ 9.6, $1.7 \mathrm{~Hz}, 1 \mathrm{H}), 6.18(\mathrm{dd}, J=9.6,4.0 \mathrm{~Hz}, 1 \mathrm{H}), 4.97$ (d, $J=5.6 \mathrm{~Hz}, 1 \mathrm{H}), 3.93 \sim 3.90(\mathrm{~m}, 1 \mathrm{H}), 1.62(\mathrm{~s}, 1 \mathrm{H}) ;{ }^{13} \mathrm{C}$ NMR $\left(125 \mathrm{MHz}, \mathrm{CDCl}_{3}\right) \delta: 137.7,136.1,132.6,129.6$, $129.2,128.6,128.3,128.2,128.0,127.4,126.7,126.3$, 71.3, 47.3; HRMS (APCI-ion trap) calcd for $\mathrm{C}_{16} \mathrm{H}_{12} \mathrm{O}$ $[\mathrm{M}-2 \mathrm{H}]^{-} 220.0899$, found 220.0889 .

$\left(1 S^{*}, 2 R^{*}\right)$-2-(4-Methylphenyl)-1,2-dihydronaphthalen1-ol (3b): Colorless oil (44.4 mg, 94\% yield). $R_{\mathrm{f}}=0.20$ on silica gel (ethyl acetate/petroleum ether, $V: V=1: 20$ ). The ee was determined to be $62 \%$ using HPLC analysis on a chiralcel OD-H column (hexane/2-propanol, $V: V=$ $99: 1,1.0 \mathrm{~mL} / \mathrm{min}, \lambda=254 \mathrm{~nm})$. Retention times were 15.346 (major) and 31.153 (minor) $\mathrm{min} .{ }^{1} \mathrm{H}$ NMR (500 $\left.\mathrm{MHz}, \mathrm{CDCl}_{3}\right) \delta: 7.39 \sim 7.15(\mathrm{~m}, 8 \mathrm{H}), 6.74(\mathrm{dd}, J=9.6,1.8$ $\mathrm{Hz}, 1 \mathrm{H}), 6.16(\mathrm{dd}, J=9.6,4.1 \mathrm{~Hz}, 1 \mathrm{H}), 4.96(\mathrm{t}, J=6.9 \mathrm{~Hz}$, $1 \mathrm{H}), 4.05 \sim 3.68(\mathrm{~m}, 1 \mathrm{H}), 2.37(\mathrm{~s}, 3 \mathrm{H}), 1.59(\mathrm{~s}, 1 \mathrm{H}) ;{ }^{13} \mathrm{C}$ NMR $\left(125 \mathrm{MHz}, \mathrm{CDCl}_{3}\right) \delta: 137.0,136.2,134.4,132.7$, $129.9,129.3,129.1,128.2,128.0,127.9,126.6,126.3$, 71.2, 46.9, 21.0; HRMS (APCI-ion trap) calcd for $\mathrm{C}_{17} \mathrm{H}_{15} \mathrm{O}$ $[\mathrm{M}-\mathrm{H}]^{-}$235.1123, found 235.1123.

$\left(1 S^{*}, 2 R^{*}\right)$-2-(2-Methylphenyl)-1,2-dihydronaphthalen1-ol (3c): Colorless oil ( $43.7 \mathrm{mg}, 92 \%$ yield). $R_{\mathrm{f}}=0.20$ on silica gel (ethyl acetate/petroleum ether, $V: V=1: 20$ ). The ee was determined to be $20 \%$ using HPLC analysis on a vhiralcel OD-H column (hexane/2-propanol, $V: V=$ $90: 10,1.0 \mathrm{~mL} / \mathrm{min}, \lambda=254 \mathrm{~nm})$. Retention times were 6.952 (minor) and 11.725 (major) min. ${ }^{1} \mathrm{H}$ NMR (500 $\left.\mathrm{MHz}, \mathrm{CDCl}_{3}\right) \delta: 7.53 \sim 7.16(\mathrm{~m}, 8 \mathrm{H}), 6.76(\mathrm{dd}, J=9.6,2.2$ $\mathrm{Hz}, 1 \mathrm{H}), 6.11(\mathrm{dd}, J=9.6,3.1 \mathrm{~Hz}, 1 \mathrm{H}), 4.87(\mathrm{~s}, 1 \mathrm{H})$, $4.25 \sim 4.23(\mathrm{~m}, 1 \mathrm{H}), 2.47(\mathrm{~s}, 3 \mathrm{H}), 1.56(\mathrm{~s}, 1 \mathrm{H}) ;{ }^{13} \mathrm{C} \mathrm{NMR}$ 
$\left(125 \mathrm{MHz}, \mathrm{CDCl}_{3}\right) \delta: 136.7,136.6,135.4,132.5,130.7$, $130.43129 .3,128.61127 .9$, 127.9, 127.6, 127.3, 126.5, 126.3, 69.6, 43.2, 19.8; HRMS (APCI-ion trap) calcd for $\mathrm{C}_{17} \mathrm{H}_{17} \mathrm{O}[\mathrm{M}+\mathrm{H}]^{+}$237.1279, found 327.1274.

(1 $\left.S^{*}, 2 R^{*}\right)$-2-(4-tertbutylphenyl)-1,2-dihydronaphthalen1-ol (3d): Colorless oil (52.9 mg, 95\% yield). $R_{\mathrm{f}}=0.20$ on silica gel (ethyl acetate/petroleum ether, $V: V=1: 20$ ). The ee was determined to be $36 \%$ using HPLC analysis on a chiralcel OD-H column (hexane/2-propanol, $V: V=$ $90: 10,1.0 \mathrm{~mL} / \mathrm{min}, \lambda=254 \mathrm{~nm})$. Retention times were 5.040 (major) and 10.656 (minor) min. ${ }^{1} \mathrm{H}$ NMR (500 $\left.\mathrm{MHz}, \mathrm{CDCl}_{3}\right) \delta: 7.42 \sim 7.12(\mathrm{~m}, 8 \mathrm{H}), 6.70(\mathrm{dd}, J=9.6,2.0$ $\mathrm{Hz}, 1 \mathrm{H}), 6.14(\mathrm{dd}, J=9.6,4.1 \mathrm{~Hz}, 1 \mathrm{H}), 5.01 \sim 4.82(\mathrm{~m}$, $1 \mathrm{H}), 3.87 \sim 3.85(\mathrm{~m}, 1 \mathrm{H}), 1.57(\mathrm{~s}, 1 \mathrm{H}), 1.31(\mathrm{~s}, 9 \mathrm{H}) ;{ }^{13} \mathrm{C}$ NMR $\left(125 \mathrm{MHz}, \mathrm{CDCl}_{3}\right) \delta: 150.3,136.1,134.4,132.7$, $129.9,128.9,128.2,128.0,127.9,126.7,126.3,125.6$, 71.3, 46.8, 34.4, 31.3; HRMS (APCI-ion trap) calcd for $\mathrm{C}_{20} \mathrm{H}_{22} \mathrm{ONa}[\mathrm{M}+\mathrm{Na}]^{+}$301.1569, found 301.1563.

$\left(1 S^{*}, 2 R^{*}\right)$-2-(2-Methoxylphenyl)-1,2-dihydronaphthalen1-ol (3e): Colorless oil (45.1 mg, $90 \%$ yield). $R_{\mathrm{f}}=0.25$ on silica gel (ethyl acetate/petroleum ether, $V: V=1: 20$ ). The $e e$ was determined to be $2 \%$ using HPLC analysis on a chiralcel OD-H column (hexane/2-propanol, $V: V=90$ : $10,1.0 \mathrm{~mL} / \mathrm{min}, \lambda=254 \mathrm{~nm})$. Retention times were 7.753 (major) and 12.228 (minor) min. ${ }^{1} \mathrm{H}$ NMR (400 MHz, $\left.\mathrm{CDCl}_{3}\right) \delta: 7.55 \sim 7.04(\mathrm{~m}, 8 \mathrm{H}), 6.71(\mathrm{dd}, J=9.6,2.5 \mathrm{~Hz}$, $1 \mathrm{H}), 6.07(\mathrm{dd}, J=9.6,3.2 \mathrm{~Hz}, 1 \mathrm{H}), 4.82(\mathrm{t}, J=5.9 \mathrm{~Hz}$, $1 \mathrm{H}), 4.20(\mathrm{dt}, J=5.6,2.9 \mathrm{~Hz}, 1 \mathrm{H}), 2.43(\mathrm{~s}, 3 \mathrm{H}), 1.53$ (s, $1 \mathrm{H}) ;{ }^{13} \mathrm{C}$ NMR $\left(100 \mathrm{MHz}, \mathrm{CDCl}_{3}\right) \delta: 136.7,136.6,135.4$, $132.5,130.6,130.4,129.3,128.6,127.9,127.9,127.6$, 127.2, 126.5, 126.3, 69.6, 43.2, 19.7; HRMS (APCI-ion trap) calcd for $\mathrm{C}_{17} \mathrm{H}_{15} \mathrm{O}_{2}[\mathrm{M}-\mathrm{H}]^{-}$251.1072, found 251.1071 .

$\left(1 S^{*}, 2 R^{*}\right)$-2-(3-Methoxylphenyl)-1,2-dihydronaphthalen1-ol (3f): Colorless oil (45.9 mg, 91\% yield). $R_{\mathrm{f}}=0.25$ on silica gel (ethyl acetate/petroleum ether, $V: V=1: 20$ ). The ee was determined to be $56 \%$ using HPLC analysis on a chiralcel OD-H column (hexane/2-propanol, $V: V=$ $90: 10,1.0 \mathrm{~mL} / \mathrm{min}, \lambda=254 \mathrm{~nm})$. Retention times were 9.644 (major) and 14.246 (minor) min. ${ }^{1} \mathrm{H}$ NMR (400 $\left.\mathrm{MHz}, \mathrm{CDCl}_{3}\right) \delta: 7.36(\mathrm{~d}, J=6.7 \mathrm{~Hz}, 1 \mathrm{H}), 7.33 \sim 7.21(\mathrm{~m}$, $3 \mathrm{H}), 7.17(\mathrm{dd}, J=7.2,1.4 \mathrm{~Hz}, 1 \mathrm{H}), 6.94 \sim 6.84(\mathrm{~m}, 1 \mathrm{H})$, $6.84 \sim 6.81(\mathrm{~m}, 2 \mathrm{H}), 6.70(\mathrm{dd}, J=9.6,2.0 \mathrm{~Hz}, 1 \mathrm{H}), 6.12$ $(\mathrm{dd}, J=9.6,4.0 \mathrm{~Hz}, 1 \mathrm{H}), 4.94(\mathrm{~d}, J=5.9 \mathrm{~Hz}, 1 \mathrm{H}), 3.92 \sim$ $3.80(\mathrm{~m}, 1 \mathrm{H}), 3.74(\mathrm{~s}, 3 \mathrm{H}), 1.61(\mathrm{~s}, 1 \mathrm{H}) ;{ }^{13} \mathrm{C}$ NMR $(100$ $\left.\mathrm{MHz}, \mathrm{CDCl}_{3}\right) \delta: 159.8,139.3,136.1,132.6,129.6,129.5$, $128.3,128.2,128.0,126.7,126.4,121.5,114.8,112.9$, 71.3, 55.1, 47.3; HRMS (APCI-ion trap) calcd for $\mathrm{C}_{17} \mathrm{H}_{15} \mathrm{O}_{2}[\mathrm{M}-\mathrm{H}]^{-}$251.1072, found 251.1077.

$\left(1 S^{*}, 2 R^{*}\right)$-2-(4-Methoxylphenyl)-1,2-dihydronaphthalen1-ol (3g): Colorless oil (49.4 mg, 98\% yield). $R_{\mathrm{f}}=0.25$ on silica gel (ethyl acetate/petroleum ether, $V: V=1: 20$ ). The ee was determined to be $51 \%$ using HPLC analysis on a Chiralcel OD-H column (hexane/2-propanol, $V: V=$ $90: 10,1.0 \mathrm{~mL} / \mathrm{min}, \lambda=254 \mathrm{~nm})$. Retention times were 9.063 (major) and 14.563 (minor) min. ${ }^{1} \mathrm{H}$ NMR (400 $\left.\mathrm{MHz}, \mathrm{CDCl}_{3}\right) \delta: 7.34(\mathrm{dd}, J=4.3,3.6 \mathrm{~Hz}, 1 \mathrm{H}), 7.30 \sim 7.20$ $(\mathrm{m}, 2 \mathrm{H}), 7.21 \sim 7.10(\mathrm{~m}, 3 \mathrm{H}), 6.95 \sim 6.74(\mathrm{~m}, 2 \mathrm{H}), 6.67$ $(\mathrm{dd}, J=9.6,1.9 \mathrm{~Hz}, 1 \mathrm{H}), 6.09(\mathrm{dd}, J=9.6,4.3 \mathrm{~Hz}, 1 \mathrm{H})$, $4.92(\mathrm{t}, J=6.7 \mathrm{~Hz}, 1 \mathrm{H}), 3.82 \sim 3.78(\mathrm{~m}, 1 \mathrm{H}), 3.76(\mathrm{~s}, 3 \mathrm{H})$, $1.50(\mathrm{~s}, 1 \mathrm{H}) ;{ }^{13} \mathrm{C}$ NMR $\left(100 \mathrm{MHz}, \mathrm{CDCl}_{3}\right) \delta: 159.0,136.3$, $132.7,130.3,130.0,129.1,128.1,128.0,127.9,126.5$, 126.2, 114.1, 71.2, 55.2, 46.4; HRMS (APCI-ion trap) calcd for $\mathrm{C}_{17} \mathrm{H}_{15} \mathrm{O}_{2}[\mathrm{M}-\mathrm{H}]^{-}$251.1072, found 251.1074.

(1 $\left.S^{*}, 2 R^{*}\right)$-2-(4-Bromophenyl)-1,2-dihydronaphthalen-1ol (3h): Colorless oil (50.4 mg, 84\% yield). $R_{\mathrm{f}}=0.20$ on silica gel (ethyl acetate/petroleum ether, $V: V=1: 20$ ). The ee was determined to be $64 \%$ using HPLC analysis on a chiralcel OD-H column (hexane/2-propanol, $V: V=$ $90: 10,1.0 \mathrm{~mL} / \mathrm{min}, \lambda=254 \mathrm{~nm})$. Retention times were 7.446 (major) and 11.562 (minor) min. ${ }^{1} \mathrm{H}$ NMR (500 $\left.\mathrm{MHz}, \mathrm{CDCl}_{3}\right) \delta: 7.43(\mathrm{~d}, J=8.3 \mathrm{~Hz}, 2 \mathrm{H}), 7.32$ (dd, $J=$ 13.6, $7.2 \mathrm{~Hz}, 2 \mathrm{H}), 7.26(\mathrm{t}, J=6.9 \mathrm{~Hz}, 1 \mathrm{H}), 7.18(\mathrm{~d}, J=7.2$ $\mathrm{Hz}, 1 \mathrm{H}), 7.13(\mathrm{~d}, J=8.3 \mathrm{~Hz}, 2 \mathrm{H}), 6.71(\mathrm{dd}, J=9.6,1.5 \mathrm{~Hz}$, $1 \mathrm{H}), 6.08(\mathrm{dd}, J=9.6,4.0 \mathrm{~Hz}, 1 \mathrm{H}), 4.91(\mathrm{~s}, 1 \mathrm{H}), 3.82 \sim$ $3.80(\mathrm{~m}, 1 \mathrm{H}), 1.54(\mathrm{~s}, 1 \mathrm{H}) ;{ }^{13} \mathrm{C} \mathrm{NMR}\left(125 \mathrm{MHz}, \mathrm{CDCl}_{3}\right) \delta$ : $136.9,135.9,132.4,131.6,131.0,129.1,128.5,128.4$, 128.2, 126.5, 126.4, 121.3, 71.1, 46.7; HRMS (APCI-ion trap) calcd for $\mathrm{C}_{16} \mathrm{H}_{12} \mathrm{BrO}[\mathrm{M}-\mathrm{H}]^{-}$299.0072, found 298.9905, 299.0066.

$\left(1 S^{*}, 2 R^{*}\right)$-2-(4-Fluorophenyl)-1,2-dihydronaphthalen-1ol (3i): White solid (40.4 mg, 84\% yield). m.p. 59 $60{ }^{\circ} \mathrm{C}$. $R_{\mathrm{f}}=0.13$ on silica gel (ethyl acetate/petroleum ether, $V$ : $V=1: 20$ ). The ee was determined to be $60 \%$ using HPLC analysis on a Chiralcel OD-H column (hexane/2-propanol, $V: V=90: 10,1.0 \mathrm{~mL} / \mathrm{min}, \lambda=254 \mathrm{~nm})$. Retention times were 6.792 (major) and 11.332 (minor) min. ${ }^{1} \mathrm{H}$ NMR (400 $\left.\mathrm{MHz}, \mathrm{CDCl}_{3}\right) \delta: 7.42 \sim 7.14(\mathrm{~m}, 6 \mathrm{H}), 7.06 \sim 6.95(\mathrm{~m}, 2 \mathrm{H})$, $6.70(\mathrm{dd}, J=9.6,2.0 \mathrm{~Hz}, 1 \mathrm{H}), 6.09(\mathrm{dd}, J=9.6,4.1 \mathrm{~Hz}$, $1 \mathrm{H}), 4.92(\mathrm{~d}, J=5.8 \mathrm{~Hz}, 1 \mathrm{H}), 3.86 \sim 3.83(\mathrm{~m}, 1 \mathrm{H}), 1.59(\mathrm{~s}$, $1 \mathrm{H}) ;{ }^{13} \mathrm{C}$ NMR $\left(100 \mathrm{MHz}, \mathrm{CDCl}_{3}\right) \delta: 163.4,161.0,136.0$, $133.32,133.29,132.5,130.8,130.7,129.6,128.4,128.3$, $128.1,126.5,126.4,115.5,115.3,71.3,46.5 ;{ }^{19} \mathrm{~F}$ NMR $\left(376 \mathrm{MHz}, \mathrm{CDCl}_{3}\right) \delta$ : -115.4 ; HRMS (APCI-ion trap) calcd for $\mathrm{C}_{16} \mathrm{H}_{12} \mathrm{FO}[\mathrm{M}-\mathrm{H}]^{-}$239.0872, found 239.0873.

$\left(1 S^{*}, 2 R^{*}\right)-2-M e t h y l-1,2-d i h y d r o n a p h t h a l e n-1-o l \quad(3 \mathbf{j})$ : Colorless oil $\left(25.9 \mathrm{mg}, 81 \%\right.$ yield). $R_{\mathrm{f}}=0.21$ on silica gel (ethyl acetate/petroleum ether, $V: V=1: 20$ ). The ee was determined to be $56 \%$ using HPLC analysis on a Chiralcel AD-H column (hexane/2-propanol, $V: V=90: 10,1.0$ $\mathrm{mL} / \mathrm{min}, \lambda=254 \mathrm{~nm}$ ). Retention times were 6.494 (minor) and 6.841 (major) min. ${ }^{1} \mathrm{H}$ NMR $\left(400 \mathrm{MHz}, \mathrm{CDCl}_{3}\right) \delta$ : $7.36(\mathrm{dd}, J=7.2,1.4 \mathrm{~Hz}, 1 \mathrm{H}), 7.32 \sim 7.20(\mathrm{~m}, 2 \mathrm{H}), 7.12$ (dd, $J=7.1,1.5 \mathrm{~Hz}, 1 \mathrm{H}), 6.51(\mathrm{dd}, J=9.5,2.4 \mathrm{~Hz}, 1 \mathrm{H})$, $5.80(\mathrm{dd}, J=9.5,3.1 \mathrm{~Hz} 1 \mathrm{H}), 4.84 \sim 4.22(\mathrm{~m}, 1 \mathrm{H}), 2.79 \sim$ $2.50(\mathrm{~m}, 1 \mathrm{H}), 1.59(\mathrm{~s}, 1 \mathrm{H}), 1.25(\mathrm{~d}, J=7.4 \mathrm{~Hz}, 1 \mathrm{H}) ;{ }^{13} \mathrm{C}$ NMR $\left(100 \mathrm{MHz}, \mathrm{CDCl}_{3}\right) \delta: 136.6,132.4,132.3,128.4$, 127.6, 127.3, 126.5, 126.4, 71.6, 35.2, 14.0; HRMS (APCI-ion trap) calcd for $\mathrm{C}_{11} \mathrm{H}_{13} \mathrm{O}[\mathrm{M}+\mathrm{H}]^{-}$161.0966, found 161.0961 .

$\left(1 S^{*}, 2 R^{*}\right)-2-T h i e n y l-1,2-d i h y d r o n a p h t h a l e n-1-o l \quad(3 \mathbf{k})$ : Colorless oil ( $43.3 \mathrm{mg}, 95 \%$ yield). $R_{\mathrm{f}}=0.25$ on silica gel (ethyl acetate/petroleum ether, $V: V=1: 20$ ). The ee was determined to be $71 \%$ using HPLC analysis on a chiralcel 
OD-H column (hexane/2-propanol, $V: V=90: 10,1.0$ $\mathrm{mL} / \mathrm{min}, \lambda=254 \mathrm{~nm}$ ). Retention times were 8.842 (major) and 11.989 (minor) min. ${ }^{1} \mathrm{H}$ NMR $\left(500 \mathrm{MHz}, \mathrm{CDCl}_{3}\right) \delta$ : $7.40(\mathrm{~d}, J=7.0 \mathrm{~Hz}, 1 \mathrm{H}), 7.34 \sim 7.23(\mathrm{~m}, 2 \mathrm{H}), 7.17$ (dd, $J=$ 5.9, $1.8 \mathrm{~Hz}, 2 \mathrm{H}), 6.97$ (dd, $J=4.3,3.1 \mathrm{~Hz}, 2 \mathrm{H}), 6.65$ (dd, $J=9.6,0.9 \mathrm{~Hz}, 1 \mathrm{H}), 6.16(\mathrm{dd}, J=9.5,4.6 \mathrm{~Hz}, 1 \mathrm{H}), 5.01(\mathrm{t}$, $J=6.5 \mathrm{~Hz}, 1 \mathrm{H}), 4.34 \sim 3.95(\mathrm{~m}, 1 \mathrm{H}), 1.86(\mathrm{~s}, 1 \mathrm{H}) ;{ }^{13} \mathrm{C}$ NMR $\left(125 \mathrm{MHz}, \mathrm{CDCl}_{3}\right) \delta: 139.9,136.2,132.4,129.5$, $128.18,128.15,128.0,126.9,126.4,126.4,126.2$, 125.1, 71.0, 42.5; HRMS (APCI-ion trap) calcd for $\mathrm{C}_{14} \mathrm{H}_{11} \mathrm{OS}$ $[\mathrm{M}-\mathrm{H}]^{-}$227.0531, found 227.0531.

$\left(1 S^{*}, 2 R^{*}\right)$-5,8-Dimethoxy-2-cyclopropyl-1,2-dihydronaphthalen-1-ol (4a): Colorless oil (44.3 mg, 90\% yield). $R_{\mathrm{f}}=0.2$ on silica gel (ethyl acetate/petroleum ether, $V$ : $V=1: 10$ ). The ee was determined to be $97 \%$ using HPLC analysis on a chiralcel OD-H column (hexane/2-propanol, $V: V=90: 10,1.0 \mathrm{~mL} / \mathrm{min}, \lambda=254 \mathrm{~nm})$. Retention times were 11.343 (minor) and 18.273 (major) min. ${ }^{1} \mathrm{H}$ NMR $\left(400 \mathrm{MHz}, \mathrm{CDCl}_{3}\right) \delta: 6.91(\mathrm{dd}, J=9.8,3.2 \mathrm{~Hz}, 1 \mathrm{H}), 6.77$ $(\mathrm{d}, J=3.4 \mathrm{~Hz}, 2 \mathrm{H}), 6.01 \sim 5.94(\mathrm{~m}, 1 \mathrm{H}), 5.04(\mathrm{~s}, 1 \mathrm{H}), 3.84$ (s, 3H), 3.79 (s, 3H), $1.77(\mathrm{~d}, J=6.3 \mathrm{~Hz}, 1 \mathrm{H}), 1.57 \sim 1.52$ $(\mathrm{m}, 1 \mathrm{H}), 1.32 \sim 1.14(\mathrm{~m}, 2 \mathrm{H}), 0.47(\mathrm{dd}, J=10.1,6.9 \mathrm{~Hz}$, $1 \mathrm{H}), 0.39 \sim 0.17(\mathrm{~m}, 2 \mathrm{H}) ;{ }^{13} \mathrm{C} \mathrm{NMR}\left(100 \mathrm{MHz} \mathrm{CDCl}_{3}\right) \delta$ : $150.6,149.6,130.3,125.4,122.8,120.8,111.3,110.6$, 63.4, 56.2, 56.1, 46.2, 11.1, 3.9, 3.7; HRMS (ESI-ion trap) calcd for $\mathrm{C}_{15} \mathrm{H}_{17} \mathrm{O}_{2}\left[\mathrm{M}+\mathrm{H}-\mathrm{H}_{2} \mathrm{O}\right]^{+}$229.1223, found 229.1216.

$\left(1 S^{*}, 2 R^{*}\right)-5,8$-Dimethoxy-2-(4-Fluorophenyl)-1,2-dihydronaphthalen-1-ol (4b): Colorless oil (50.0 mg, 85\% yield). $R_{\mathrm{f}}=0.2$ on silica gel (ethyl acetate/petroleum ether, $V: V=1: 10)$. The ee was determined to be $52 \%$ using HPLC analysis on a chiralcel OD-H column (hexane/ 2-propanol, $V: V=90: 10,1.0 \mathrm{~mL} / \mathrm{min}, \lambda=254 \mathrm{~nm})$. Retention times were 17.871 (major) and 32.805 (minor) min. ${ }^{1} \mathrm{H}$ NMR $\left(400 \mathrm{MHz}, \mathrm{CDCl}_{3}\right) \delta: 7.39(\mathrm{dd}, J=8.6,5.4$ $\mathrm{Hz}, 1 \mathrm{H}), 7.19 \sim 6.98(\mathrm{~m}, 1 \mathrm{H}), 6.83(\mathrm{q}, J=9.0 \mathrm{~Hz}, 1 \mathrm{H})$, $6.16 \sim 5.95(\mathrm{~m}, 1 \mathrm{H}), 5.06(\mathrm{dd}, J=4.7,1.5 \mathrm{~Hz}, 1 \mathrm{H}), 3.83(\mathrm{~d}$, $J=5.1 \mathrm{~Hz}, 1 \mathrm{H}) ;{ }^{13} \mathrm{C} \mathrm{NMR}\left(100 \mathrm{MHz}, \mathrm{CDCl}_{3}\right) \delta: 163.2$, $160.8,150.7,149.7,136.3,136.2,130.7,130.6,128.8$, 122.2, 115.4, 115.2, 111.5, 111.1, 64.4, 56.2, 46.5, 22.6; ${ }^{19} \mathrm{~F}$ NMR $\left(375 \mathrm{MHz}, \mathrm{CDCl}_{3}\right) \delta$ : -116.10 ; HRMS (ESIion trap) calcd for $\mathrm{C}_{18} \mathrm{H}_{16} \mathrm{O}_{2} \mathrm{~F}\left[\mathrm{M}+\mathrm{H}-\mathrm{H}_{2} \mathrm{O}\right]^{+}$283.1129, found 283.1120 .

$\left(1 S^{*}, 2 R^{*}\right)-5,8$-Dimethoxy-2-(4-Methylphenyl)-1,2-dihydronaphthalen-1-ol (4c): Colorless oil (53.9 mg, 91\% yield). $R_{\mathrm{f}}=0.2$ on silica gel (ethyl acetate/petroleum ether, $V: V=1: 10$ ). The ee was determined to be $51 \%$ using HPLC analysis on a Chiralcel OD-H column (hexane/2-propanol, $V: V=90: 10,1.0 \mathrm{~mL} / \mathrm{min}, \lambda=254 \mathrm{~nm})$. Retention times were 15.041 (major) and 25.944 (minor) min. ${ }^{1} \mathrm{H}$ NMR $\left(400 \mathrm{MHz}, \mathrm{CDCl}_{3}\right) \delta: 7.34(\mathrm{~d}, J=8.0 \mathrm{~Hz}$, 2H), $7.22(\mathrm{~d}, J=7.8 \mathrm{~Hz}, 2 \mathrm{H}), 7.09(\mathrm{dd}, J=9.8,3.2 \mathrm{~Hz}$, $1 \mathrm{H}), 6.82(\mathrm{q}, J=9.0 \mathrm{~Hz}, 2 \mathrm{H}), 6.16 \sim 6.12(\mathrm{~m}, 1 \mathrm{H}), 5.08$ $(\mathrm{dd}, J=4.6,1.2 \mathrm{~Hz}, 1 \mathrm{H}), 3.84(\mathrm{~s}, 1 \mathrm{H}), 3.83(\mathrm{~s}, 1 \mathrm{H}), 3.80 \sim$ $3.75(\mathrm{~m}, 1 \mathrm{H}), 2.38(\mathrm{~s}, 3 \mathrm{H}) ;{ }^{13} \mathrm{C} \mathrm{NMR}\left(100 \mathrm{MHz}, \mathrm{CDCl}_{3}\right) \delta$ : $150.8,149.7,137.2,136.5,129.2,129.1,128.9,124.4$, $122.6,121.9,111.4,110.9,64.3,56.2,56.2,46.8,21.1$;
HRMS (ESI-ion trap) calcd for $\mathrm{C}_{19} \mathrm{H}_{19} \mathrm{O}_{2}\left[\mathrm{M}+\mathrm{H}-\mathrm{H}_{2} \mathrm{O}\right]^{+}$ 279.1380 , found 279.1372 .

$\left(1 S^{*}, 2 R^{*}\right)$-2-Phenyl-1,2-dihydrotriphenylen-1-ol (5a): A white olid (54.8 mg, $85 \%$ yield). m.p. $157 \sim 158{ }^{\circ} \mathrm{C} . R_{\mathrm{f}}=$ 0.23 on silica gel (ethyl acetate/petroleum ether, $V: V=$ $1: 10)$. The ee was determined to be $55 \%$ using HPLC analysis on a chiralcel AD-H column (hexane/2-propanol, $V: V=90: 10,1.0 \mathrm{~mL} / \mathrm{min}, \lambda=254 \mathrm{~nm})$. Retention times were 46.597 (major) and 49.684 (minor) min. ${ }^{1} \mathrm{H}$ NMR $\left(500 \mathrm{MHz}, \mathrm{CDCl}_{3}\right) \delta: 8.87 \sim 8.62(\mathrm{~m}, 2 \mathrm{H}), 8.50 \sim 8.36(\mathrm{~m}$, $1 \mathrm{H}), 8.30 \sim 8.18(\mathrm{~m}, 1 \mathrm{H}), 7.78 \sim 7.69(\mathrm{~m}, 2 \mathrm{H}), 7.66(\mathrm{~d}, J=$ $9.8 \mathrm{~Hz}, 1 \mathrm{H}), 7.64 \sim 7.58(\mathrm{~m}, 2 \mathrm{H}), 7.23 \sim 7.07(\mathrm{~m}, 5 \mathrm{H})$, $6.52 \sim 6.48(\mathrm{~m}, 1 \mathrm{H}), 5.58(\mathrm{~d}, J=7.4 \mathrm{~Hz}, 1 \mathrm{H}), 4.16(\mathrm{~d}, J=$ $5.9 \mathrm{~Hz}, 1 \mathrm{H}), 2.17(\mathrm{~d}, J=7.6 \mathrm{~Hz}, 1 \mathrm{H}) ;{ }^{13} \mathrm{C} \mathrm{NMR}(126 \mathrm{MHz}$, $\left.\mathrm{CDCl}_{3}\right) \delta: 138.6,130.8,130.6,130.1,129.9,128.7,128.6$, $127.8,127.4,127.3,127.0,126.97,126.9,126.5,126.4$, 123.8, 123.6, 123.1, 123.0, 122.3, 69.4, 48.8; ${ }^{1} \mathrm{HRMS}$ (ESI-ion trap) calcd for $\mathrm{C}_{24} \mathrm{H}_{29} \mathrm{O}[\mathrm{M}+\mathrm{H}]^{+}$323.1431, found 323.1430 .

$\left(1 S^{*}, 2 R^{*}\right)$-2-Cyclopropyl-1,2-dihydrotriphenylen-1-ol (5b): Colorless oil $\left(51.5 \mathrm{mg}, 90 \%\right.$ yield). $R_{\mathrm{f}}=0.18$ on silica gel (ethyl acetate/petroleum ether, $V: V=1: 10$ ). The ee was determined to be $97 \%$ using HPLC analysis on a Chiralcel AD-H column (hexane/2-propanol, $V: V=90: 10$, $1.0 \mathrm{~mL} / \mathrm{min}, \lambda=254 \mathrm{~nm}$ ). Retention times were 25.025 (major) and 26.224 (minor) min. ${ }^{1} \mathrm{H}$ NMR (500 MHz, $\left.\mathrm{CDCl}_{3}\right) \delta: 8.82 \sim 8.69(\mathrm{~m}, 2 \mathrm{H}), 8.40(\mathrm{~d}, J=7.8 \mathrm{~Hz}, 1 \mathrm{H})$, $8.32 \sim 8.23(\mathrm{~m}, 1 \mathrm{H}), 7.78 \sim 7.58(\mathrm{~m}, 4 \mathrm{H}), 7.43(\mathrm{dd}, J=9.8$, $3.2 \mathrm{~Hz}, 1 \mathrm{H}), 6.43 \sim 6.27(\mathrm{~m}, 1 \mathrm{H}), 5.45(\mathrm{~d}, J=4.5 \mathrm{~Hz}, 1 \mathrm{H})$, $3.69(\mathrm{t}, J=6.8 \mathrm{~Hz}, 1 \mathrm{H}), 1.91 \sim 1.76(\mathrm{~m}, 1 \mathrm{H}), 0.87 \sim 0.71$ $(\mathrm{m}, 2 \mathrm{H}), 0.56 \sim 0.31(\mathrm{~m}, 2 \mathrm{H}) ;{ }^{13} \mathrm{C} \mathrm{NMR}(125 \mathrm{MHz}$, $\left.\mathrm{CDCl}_{3}\right) \delta: 132.1,130.5,130.4,129.8,129.6,128.7,127.2$, $126.8,126.7,126.7,126.3,124.1,123.7,123.0,122.9$, 122.7, 66.5, 46.8, 22.6, 11.1, 3.8; ${ }^{1}$ HRMS (ESI-ion trap) calcd for $\mathrm{C}_{21} \mathrm{H}_{17}[\mathrm{M}+\mathrm{H}]^{+}$269.1325, found 269.1320.

$\left(1 S^{*}, 2 R^{*}\right)$-2-(4-Fluorophenyl)-1,2-dihydrotriphenylen-1ol (5c): Colorless oil (57.2 mg, 84\% yield). $R_{\mathrm{f}}=0.15$ on silica gel (ethyl acetate/petroleum ether, $V: V=1: 10$ ). The ee was determined to be $68 \%$ using HPLC analysis on a Chiralcel AD-H column (hexane/2-propanol, $V: V=$ $90: 10,1.0 \mathrm{~mL} / \mathrm{min}, \lambda=254 \mathrm{~nm})$. Retention times were 37.883 (major) and 67.269 (minor) $\mathrm{min} .{ }^{1} \mathrm{H}$ NMR (500 $\left.\mathrm{MHz}, \mathrm{CDCl}_{3}\right) \delta: 8.98 \sim 8.63(\mathrm{~m}, 2 \mathrm{H}), 8.40 \sim 8.29(\mathrm{~m}, 1 \mathrm{H})$, $8.29 \sim 8.20(\mathrm{~m}, 1 \mathrm{H}), 7.78 \sim 7.62(\mathrm{~m}, 4 \mathrm{H}), 7.59(\mathrm{dd}, J=9.9$, $3.3 \mathrm{~Hz}, 1 \mathrm{H}), 7.50(\mathrm{dd}, J=8.5,5.4 \mathrm{~Hz}, 2 \mathrm{H}), 7.16(\mathrm{t}, J=8.7$ $\mathrm{Hz}, 2 \mathrm{H}), 6.44 \sim 6.41(\mathrm{~m}, 1 \mathrm{H}), 5.41(\mathrm{~d}, J=4.4 \mathrm{~Hz}, 1 \mathrm{H})$, $4.08 \sim 3.95(\mathrm{~m}, 1 \mathrm{H}) ;{ }^{13} \mathrm{C}$ NMR $\left(125 \mathrm{MHz}, \mathrm{CDCl}_{3}\right) \delta$ : $163.1,161.1,135.9,130.7,130.7,129.7,128.6,128.58$, $127.3,127.0,126.5,126.4,124.1,123.7,123.1,123.09$, $115.6,115.5,67.6,47.3 ;{ }^{19} \mathrm{~F}$ NMR $\left(375 \mathrm{MHz} \mathrm{CDCl}_{3}\right) \delta$ : -115.57 ; ${ }^{1} \mathrm{H}$ RMS (ESI-ion trap) calcd for $\mathrm{C}_{24} \mathrm{H}_{18} \mathrm{OF}$ $[\mathrm{M}+\mathrm{H}]^{+}$341.1336, found 341.1331.

(1 $\left.S^{*}, 2 R^{*}\right)$-2-(4-methylphenyl)-1,2-dihydrotriphenylen-1ol (5d). Colorless oil (61.2 mg, 91\% yield). $R_{\mathrm{f}}=0.20$ on silica gel (ethyl acetate/petroleum ether, $V: V=1: 10$ ). The ee was determined to be $65 \%$ using HPLC analysis on a Chiralcel AD-H column (hexane/2-propanol, $V: V=$ 
$90: 10,1.0 \mathrm{~mL} / \mathrm{min}, \lambda=254 \mathrm{~nm})$. Retention times were 38.762 (major) and 45.206 (minor) min. ${ }^{1} \mathrm{H}$ NMR (500 $\left.\mathrm{MHz}, \mathrm{CDCl}_{3}\right) \delta: 8.77 \sim 8.70(\mathrm{~m}, 2 \mathrm{H}), 8.54 \sim 8.36(\mathrm{~m}, 1 \mathrm{H})$, $8.33 \sim 8.15(\mathrm{~m}, 1 \mathrm{H}), 7.77 \sim 7.70(\mathrm{~m}, 2 \mathrm{H}), 7.66(\mathrm{~d}, J=9.8$ $\mathrm{Hz}, 1 \mathrm{H}), 7.63 \sim 7.58(\mathrm{~m}, 2 \mathrm{H}), 7.01(\mathrm{dd}, J=13.5,5.7 \mathrm{~Hz}$, $2 \mathrm{H}), 6.97 \sim 6.90(\mathrm{~m}, 2 \mathrm{H}), 6.65 \sim 6.33(\mathrm{~m}, 1 \mathrm{H}), 5.58(\mathrm{~s}$, $1 \mathrm{H}), 4.13(\mathrm{~d}, J=4.6 \mathrm{~Hz}, 1 \mathrm{H}), 2.20(\mathrm{~s}, 3 \mathrm{H}) ;{ }^{13} \mathrm{C}$ NMR $(125$ $\left.\mathrm{MHz}, \mathrm{CDCl}_{3}\right) \delta: 138.7,138.3,130.8,130.6,130.1,129.9$, $128.7,128.5,127.8,127.4,127.3,127.0,126.9,126.4$, $126.4,124.6,123.9,123.6,123.1,123.0,122.2,69.5,48.8$, 21.4; ${ }^{1} \mathrm{HRMS}$ (ESI-ion trap) calcd for $\mathrm{C}_{25} \mathrm{H}_{20} \mathrm{ONa}[\mathrm{M}+$ $\mathrm{Na}]^{+}$359.1406, found 359.1401.

$\left(1 S^{*}, 2 R^{*}\right)$-2-Thienyl-1,2-dihydrotriphenylen-1-ol (5e): Colorless oil $\left(61.0 \mathrm{mg}, 93 \%\right.$ yield). $R_{\mathrm{f}}=0.20$ on silica gel (ethyl acetate/petroleum ether, $V: V=1: 10$ ). The $e e$ was determined to be $87 \%$ using HPLC analysis on a Chiralcel AD-H column (hexane/2-propanol, $V: V=90: 10,1.0$ $\mathrm{mL} / \mathrm{min}, \lambda=254 \mathrm{~nm}$ ). Retention times were 55.292 (major) and 58.044 (minor) min. ${ }^{1} \mathrm{H}$ NMR $\left(500 \mathrm{MHz}, \mathrm{CDCl}_{3}\right) \delta$ : $9.01 \sim 8.60(\mathrm{~m}, 2 \mathrm{H}), 8.48 \sim 8.23(\mathrm{~m}, 2 \mathrm{H}), 7.93 \sim 7.64(\mathrm{~m}$, $4 \mathrm{H}), 7.59(\mathrm{dd}, J=9.8,3.3 \mathrm{~Hz}, 1 \mathrm{H}), 7.46 \sim 7.33(\mathrm{~m}, 1 \mathrm{H})$, $7.27(\mathrm{~d}, J=3.4 \mathrm{~Hz}, 1 \mathrm{H}), 7.15(\mathrm{dd}, J=5.2,3.4 \mathrm{~Hz}, 1 \mathrm{H})$, $6.52 \sim 6.34(\mathrm{~m}, 1 \mathrm{H}), 5.53(\mathrm{~d}, J=4.2 \mathrm{~Hz}, 1 \mathrm{H}), 4.47 \sim 4.23$ $(\mathrm{m}, 1 \mathrm{H}) ;{ }^{13} \mathrm{C}$ NMR $\left(125 \mathrm{MHz}, \mathrm{CDCl}_{3}\right): \delta 142.7,130.7$, $130.66,130.6,129.7,128.9,128.6,127.4,127.1,127.0$, $126.0,126.4,126.1,124.8,124.1,124.0,123.9,123.1$, 123.1, 67.7, 43.5; ${ }^{1}$ HRMS (ESI-ion trap) calcd for $\mathrm{C}_{22} \mathrm{H}_{15} \mathrm{~S}$ $\left[\mathrm{M}-\mathrm{H}_{2} \mathrm{O}+\mathrm{H}\right]^{+}$311.0889, found 311.0885.

Supporting Information Copies of ${ }^{13} \mathrm{C}$ NMR and ${ }^{1} \mathrm{H}$ NMR and ${ }^{19} \mathrm{~F}$ NMR spectra for all products, HRMS and HPLC data of all products, crystal structure and data of the product 3d. The Supporting Information is available free of charge via the Internet at http://sioc-journal.cn.

\section{References}

[1] (a) Roberto, P.; Francesco, B.; Nicola, C. A.; Marcello, L.; Vincenzo, T.; Francesco, F.; Vincenzo, O.; Alberto, G.; Stefano, G. J. Med. Chem. 1995, 38, 942.

(b) Scott, E. S.; Felix, A. A.; Ratna, C.; David, E. N.; Val, J. W.; Richard, B. M. J. Med. Chem. 1995, 38, 2395.

(c) Lautens, M.; Rovis, T. J. Org. Chem. 1997, 62, 5246.

(d) Lautens, M.; Rovis, T. J. Am. Chem. Soc. 1997, 119, 11090.

(e) Lautens, M.; Fagnou, K.; Taylor, M.; Rovis, T. J. Organomet. Chem. 2001, 624, 259

(f) Lautens, M.; Fagnou, K.; Zunic, V. Org. Lett. 2002, 4, 3465.

(g) Tsoung, J.; Krämer, K.; Zajdlik, A.; Liébert, C.; Lautens, M. J. Org. Chem. 2011, 76, 9031.

[2] For reviews, see: (a) Fagnou, K.; Lautens, M. Chem. Rev. 2003, 103, 169.

(b) Hayashi, T.; Yamasaki, K. Chem. Rev. 2003, 103, 2829.

(c) Lautens, M.; Fagnou, K.; Hiebert, S. Acc. Chem. Res. 2003, 36, 48.

(d) Rayabarapu, D. K.; Cheng, C.-H. Acc. Chem. Res. 2007, 40, 971.

[3] Nakamura, M.; Matsuo, K.; Inoue, T.; Nakamura, E. Org. Lett. 2003, 5, 1373 .

[4] (a) Lautens, M.; Ma, S. J. Org. Chem. 1996, 61, 7246. (b) Rayabarapu, D. K.; Chiou, C.-F.; Cheng, C.-H. Org. Lett. 2002, 4,1679 . (c) Wang, G.; Gan, Y.; Liu, Y. Chin. J. Chem. 2018, 36, 916.

(d) Fu, X.-P.; Xiao, Y.-L.; Zhang, X. Chin. J. Chem. 2018, 36, 143.

[5] (a) Bertozzi, F.; Pineschi, M.; Macchia, F.; Arnold, L. A.; Minnaard, A. J.; Feringa, B. L. Org. Lett. 2002, 4, 2703.

(b) Zhang, W.; Wang, L.-X.; Shi, W.-J.; Zhou, Q.-L. J. Org. Chem. 2005, 70, 3734.

(c) Zhang, L.-Y.; Wang, L. Chin. J. Chem. 2006, 24, 1605.

[6] (a) Carreras, J.; Avenoza, A.; Busto, J. H.; Peregrina, J. M. Org. Lett. 2007, 9, 1235.

(b) Cortez, G. A.; Baxter, C. A.; Schrock, R. R.; Hoveyda, A. H. Org. Lett. 2007, 9, 2871.

(c) Machin, B. P.; Howell, J.; Mandel, J.; Blanchard, N.; Tam, W. Org. Lett. 2009, 11, 2077.

[7] (a) Lautens, M.; Fagnou, K.; Taylor, M. Org. Lett. 2000, 2, 1677.

(b) Lautens, M.; Fagnou, K. Tetrahedron 2001, 57, 5067.

(c) Lautens, M.; Fagnou, K. J. Am. Chem. Soc. 2001, 123, 7170.

(d) Preetz, A.; Kohrt, C.; Drexler, H. J.; Torrens, A.; Buschmann, H.; Lopez, M. G.; Heller, D. Adv. Synth. Catal. 2010, 352, 2073.

[8] (a) Lautens, M.; Renaud, J.-L.; Hiebert, S. J. Am. Chem. Soc. 2000, $122,1804$.

(b) Li, L.-P.; Rayabarapu, D. K.; Nandi, M.; Cheng, C.-H. Org. Lett. 2003, 5, 1621.

(c) Cabrera, S.; Arrayás, R. G.; Carretero, J. C. Angew. Chem., Int. Ed. 2004, 43, 3944.

(d) Ogura, T.; Yoshida, K.; Yanagisawa, A.; Imamoto, T. Org. Lett. 2009, 11, 2245.

(e) Li, M.; Yan, X.-X.; Hong, W.; Zhu, X.-Z.; Cao, B.-X.; Sun, J.; Hou, X.-L. Org. Lett. 2004, 6, 2833.

[9] (a) Pan, X.-J.; Huang, G.-B.; Long, Y.-H.; Zuo, X.-J.; Xu, X.; Gu, F.-L.; Yang, D.-Q. J. Org. Chem. 2014, 79, 187.

(b) Yang, D.-Q.; Liang, N. Org. Biomol. Chem. 2014, 12, 2080.

(c) Meng, L.; Yang, W.; Pan, X.-J.; Tao, M.; Cheng, G.; Wang, S.-Y.; Zeng, H.-P.; Long, Y.-H.; Yang, D.-Q. J. Org. Chem. 2015, $80,2503$.

[10] (a) Yang, D.-Q.; Long, Y.-H.; Wang, H.; Zhang, Z.-M. Org. Lett. 2008, 10, 4723.

(b) Long, Y.-H.; Yang, D.-Q.; Zhang, Z.-M.; Wu, Y.-J.; Zeng, H.-P.; Chen, Y. J. Org. Chem. 2010, 75, 7291.

(c) Yang, D.-Q.; Long, Y.-H.; Zhang, J.-F.; Zeng, H.-P.; Wang, S.-Y.; Li, C.-R. Organometallics 2010, 29, 3477.

(d) Yang, D.-Q.; Long, Y.-H.; Wu, Y.-J.; Zuo, X.-J.; Tu, Q.-Q.; Fang, S.; Jiang, L.-S.; Wang, S.-Y.; Li, C.-R. Organometallics 2010, $29,5936$.

(e) Fang, S.; Liang, X.-L.; Long, Y.-H.; Li, X.-L.; Yang, D.-Q.; Wang, S.-Y.; Li, C.-R. Organometallics 2012, 31, 3113.

(f) Cheng, H.-C.; Yang, D.-Q. J. Org. Chem. 2012, 77, 9756.

(g) Yang, D.-Q.; Xia, J.-Y.; Long, Y.-H.; Zeng, Z.-Y.; Zuo, X.-J.; Wang, S.-Y.; Li, C.-R. Org. Biomol. Chem. 2013, 11, 4871.

(h) Yang, W.; Luo, R.-S.; Yang, D.-Q. Molecules 2015, 20, 21103.

[11] (a) Feng, C.-C.; Nandi, M.; Sambaiah, T.; Cheng, C.-H. J. Org. Chem. 1999, 64, 3538.

(b) Chen, C.-L.; Martin, S. F. Org. Lett. 2004, 6, 3581.

(c) Chen, C.-L.; Martin, S.-F. J. Org. Chem. 2006, 71, 4810.

[12] (a) Arrayas, R. G.; Cabrera, S.; Carretero, J. C. Org. Lett. 2003, 5, 1333.

(b) Zhang, W.; Zhu, S.-F.; Qiao, X.-C.; Zhou, Q.-L. Chem.-Asian J. 2008, 3, 2105 .

[13] (a) Lautens, M.; Dockendorff, C.; Fagnou, K.; Malicki, A. Org. Lett. 2002, 4, 1311.

(b) Murakami, M.; Igawa, H. Chem. Commun. 2002, 390.

(c) Lautens, M.; Dockendorff, C. Org. Lett. 2003, 5, 3695.

(d) Zhang, T.-K.; Mo, D.-L.; Dai, L.-X.; Hou, X.-L. Org. Lett. 2008, 10, 3689.

(d) Luo, F.; Pan, C.; Cheng, J. Chin. J. Org. Chem. 2010, 30, 633.

[14] (a) Li, Y.; Yang, W.; Cheng, G.; Yang, D.-Q. J. Org. Chem. 2016, 81,4744 .

(b) Wu, R. H.; Yang, W.; Chen, W. K.; Yang, D.-Q. Org. Chem. Front. 2017, 4, 1921. 
(c) Zhang, S.; Yang, S.; Huang, L.; Zhao, B.; Cheng, K.; Qi, C. Chin. J. Org. Chem. 2015, 35, 2259 (in Chinese).

(张诗浓, 杨胜虎, 黄乐浩, 赵保丽, 程凯, 齐陈泽, 有机化学, 2015, 35, 2259.)

[15] (a) Seigerman, C. K.; Micyus, T. M.; Neufeldt, S. R.; Sanford, M. S. Tetrahedron 2013, 69, 5580.

(b) Dubbaka, S. R.; Narreddula, V. R.; Gadde, S.; Mathew, T. Tetrahedron 2014, 70, 9676.

[16] Molander, G. A.; Sandrock, D. L. Curr. Opin. Drug Discovery Dev. $\mathbf{2 0 0 9}, 12,811$.

[17] (a) Li, S. F.; Xu, J. B.; Fan, B.-M.; Lu, Z. W.; Zeng, C. Y.; Bian, Z. X.; Zhou, Y. Y.; Wang, J. Chem.-Eur. J. 2015, 21, 9003. (b) Xu, X.; Chen, J.-C.; He, Z.-X.; Zhou, Y.-Y.; Fan, B.-M. Org. Biomol. Chem. 2016, 14, 2480.

[18] (a) Barbeiro, C. S.; Vasconcelos, S. N. S.; de Oliveira, I. M.; Zukerman-Schpector, J.; Caracelli, I.; Maganhi, S. H.; Stefani, H. A. ChemistrySelect 2017, 2, 8173.

(b) Liu, L.; Dong, Y.; Pang, B.; Ma, J. J. Org. Chem. 2014, 79, 7193.

[19] Zhang, W.; Chen, J. C.; Zeng, G. Z.; Yang, F.; Xu, J. B.; Sun, W. Q.; Shinde, M. V.; Fan, B. M. J. Org. Chem. 2017, 82, 2641.

[20] (a) Lautens, M.; Fagnou, K.; Yang, D.-Q. J. Am. Chem. Soc. 2003, 125,14884

(b) Villeneuve, K.; Tam, W. Organometallics 2007, 26, 6082.

(Zhao, C.) 